\title{
ÉDITORIAL Retour du rédacteur en chef
}

Laurence Klotz, MD, FRCSC

Rédacteur en chef

JAUC her lecteur, chère lectrice,

Je me réjouis d'avoir été invité par l'AUC à reprendre mes fonctions de rédacteur en chef du JAUC. Lors des 12 derniers mois, le $\mathrm{D}^{\mathrm{r}}$ Armen Aprikian a fait un superbe travail de rédaction pendant que j'occupais la présidence de I'AUC. L'expérience a été fort enrichissante, mais je suis très heureux d'être de retour.

Votre journal continue d'évoluer. La qualité des articles soumis s'est grandement améliorée, ce qui se reflète dans notre contenu. La publication du journal, assurée par I'AMC jusqu'à présent, sera désormais prise en charge par notre association. Ce changement nous donnera un plus grand contrôle sur la présentation et le style du journal et nous permettra aussi de stabiliser notre situation financière.

Nous prévoyons déjà un certain nombre d'autres changements. Nous présenterons des éditoriaux rédigés par des membres perspicaces et éloquents de la communauté canadienne et étrangère en urologie. Nous mettrons l'emphase sur notre section " Point/Counterpoint », qui présente les opinions d'éminents praticiens sur des sujets controversés.

Un article très intéressant du présent numéro porte sur une analyse des 100 articles les plus souvent cités dans le domaine de l'urologie, un compte rendu des plus fascinants. Comme le soulignent les auteurs, un taux élevé de citations ne se traduit pas nécessairement par une meilleure qualité ou une plus grande influence. Cela dit, il est intéressant de noter quels articles figurent sur la liste, et lesquels en sont absents. J'ai été étonné de voir que l'article le plus souvent mentionné en urologie porte sur la dysfonction érectile. Bien entendu, comme d'autres médecins canadiens sans doute, je m'attendais à y retrouver l'article de AI Morales sur le bacille Calmette-Guérin (au $51^{\mathrm{e}}$ rang). Mais il y a aussi un certain nombre d'autres articles " classiques " auxquels je fais référence depuis de nombreuses années qui ne figurent pas sur la liste, tel l'article nobélisé de Huggins et Hodges sur la castration et le cancer de la prostate (trop vieux peut-être) et le fameux article de Steve Freedman sur l'évolution naturelle des récidives biochimiques dans le cancer de la prostate. Joe Oesterling, tombé en disgrâce après avoir été condamné pour acte criminel, occupe les $13^{\mathrm{e}}$ et $78^{\mathrm{e}}$ rangs. Globalement, les 100 articles les plus souvent cités représentent une série de percées scientifiques et cliniques remarquables. Les auteurs tirent des conclusions utiles quant aux thèmes récurrents dans les études de la liste, ce qui en fait un article que vous ne voudrez pas sauter. 